\title{
Observations of the remnants of the ultrarelativistic electrons injected by the strong SSC of 24 March 1991
}

\author{
M. D. Looper and J. B. Blake \\ The Aerospace Corporation, Los Angeles, CA 90009 \\ R. A. Mewaldt and J. R. Cummings \\ California Institute of Technology, Pasadena, CA 91125 \\ D. N.. Baker \\ University of Colorado, LASP, Boulder, CO 80309
}

\begin{abstract}
Data from the SAMPEX spacecraft has shown the presence near $\mathrm{L}=1.9$ of the remnants of the ultrarelativistic electrons (above $15 \mathrm{MeV}$ ) injected into the magnetosphere on 24 March 1991. The decay of the electrons has been tracked since July 1992. The electron $e$-folding lifetime is in the range of six to twelve months, and the location in $\mathrm{L}$ of the peak intensity moved inward by $\sim 0.05$ units in one year. The inferred electron lifetime is similar to that of $\sim 0.5 \mathrm{MeV}$ electrons, which is in agreement with extrapolation to higher energies of the theoretical estimates of Lyons and Thorne (1972).
\end{abstract}

\section{Introduction}

A solar X-ray event and a 3B optical flare were observed at 2247 UT on 22 March 1991. Energetic particles, ions and electrons, began to arrive at the Earth around 0730 UT on 23 March, followed at 0342 UT on 24 March by an extremely strong and abrupt interplanetary shock wave. Immediately after the arrival of the shock, sensors aboard CRRES observed the sudden appearance of protons and electrons with energies of a few tens of $\mathrm{MeV}$, which formed a new radiation belt between $\mathrm{L}$ $=2$ and $\mathrm{L}=3.5$ (Vampola and Korth 1992; Blake et al. 1992a; Blake et al. 1992b).

In the months following the creation of this new belt, the location of the peak intensity of the injected particles was observed to move inward, the pitch-angle distribution to flatten, and the intensity to decrease with time. The CRRES satellite failed in mid-October 1991, and, thereafter, the evolution of the new radiation component could not be followed. In this Letter, we report observations of the remnants of the shockinjected electrons made at low altitude by a sensor aboard the SAMPEX (Solar, Anomalous, and Magnetospheric Particle Explorer) satellite.

\section{Instrumentation}

SAMPEX was launched into a $520 \times 670-\mathrm{km}$-altitude, $82^{\circ}$ inclination Earth orbit on 3 July 1992 (Baker et al. 1993). Its complement of four energetic-particlc instruments includes

Copyright 1994 by the American Geophysical Union.

Paper number 94GL01586

0094-8534/94/94GL-01586\$03.00
PET, the Proton/Electron Telescope (Cook et al. 1993). PET consists of a stack of $122 \mathrm{~mm}$ - to $3 \mathrm{~mm}$-thick lithium-drifted silicon detectors divided into eight functional units numbered P1 through P8 (from the aperture inward), annular guard detectors around P3 through P8, and associated electronics. The outputs of P1, P2, P3, and the sum of P4 through P7 are fed into four analog-to-digital converters (ADCs) to permit measurement of energy deposits as well as particle range. Unfortunately, the P47 ADC malfunctioned shortly after launch, but discriminators attached to the outputs of P4 to P7 still allow the ranges of individual particle events to be measured.

The telemetry stream from PET includes several singledetector and coincidence rates and a sampling of pulse-heightanalyzed events. ADC information is used to identify particle species for each event; a coincidence rate associated with each event buffer is used to normalize the counting rate of each species in the buffer, and the rate of a steady pulser is used to correct for deadtime in the PET electronics ( $c f$. Cook et al. 1993). Even in the absence of P47 ADC information it is possible to identify particle species unambiguously since protons of a given range will deposit more energy in detectors P1 to P3 than will electrons of the same range, and thus will appear as a separate track on scatterplots of energy deposited in P1 vs. P2, P2 vs. P3, etc. An exception to this is the case of penetrating particles (those triggering all detectors from P1 to P8) with low-energy deposits in P1, P2, and P3. Such particles may be either electrons or very energetic protons.

\section{Observations}

The analysis presented here was restricted to periods when the instrument was pointed within $10^{\circ}$ of perpendicular to the magnetic field, with $L$ between 1.1 and 3.9. As the SAMPEX orbit precesses, the appropriate conditions recur about every three months for a period of a month or less. Between these periods, PET looks perpendicular to the magnetic field only at $\mathrm{L}<1.2$. SAMPEX has operated continuously for over a year; we compare here five two-week periods in the middle of the first five recurrences of the appropriate attitude conditions. Electron data for the first period, 16-31 August 1992, are shown in Figure 1; these are the rates of electrons of Ranges 2 to 8, where "Range N" means that all of the detectors from P1 to PN and none of the guards around P3 to P8 were triggered. The data for each Range meeting the pitch-angle condition above were sorted by L (resolution 0.04 ) and B (resolution 0.01 gauss) and averaged over all orbits during the time period. The 


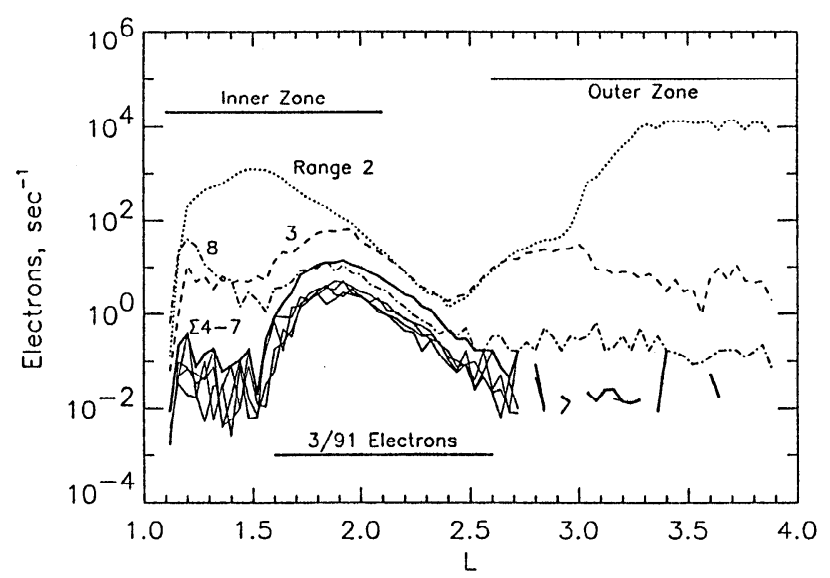

Figure 1. PET electron counting rates during 16-31 August 1992 as a function of $L$. Dotted line is Range 2, dashed is Range 3, dash-dotted is Range 8, solid lines are Ranges 4 to 7, and heavy solid line is their sum.

value plotted is the maximum average rate at each $L$, i.e., the time-averaged rate of electrons mirroring at the lowest value of B traversed at each L. Curves are labeled by their Ranges; the solid lines tangled together are for electrons of Ranges 4 through 7 (collectively referred to hereinafter as the "Deep Ranges"), whose sum is plotted as the heavy line.

The inner and outer zones of the radiation belts are distinctly visible in the Range- 2 and Range- 3 data. (The raggedness of the outer zone rates is not due to statistical fluctuations but rather to holes in the coverage of $B$ at a given (high) $L$ from day to day so that the normal variations in outer-zone electron fluxes are incompletely averaged out.) By contrast, the Deep Range electrons are concentrated around $L=1.9$. A corresponding peak can also be seen in the Range-3 data, overlapping the edge of the inner zone. The Range- 8 curve, as noted above, is contaminated by energetic protons from both galactic cosmic ray background and the heart of the inner zone, but a peak around $L=1.9$ is also visible in this Range. It is this response of the PET instrument around $L=1.9$ that we identify with the electron remnants of the distinctive radiation belt formed during the March 1991 injection.

Since the SAMPEX orbit is at low altitude, the atmospheric loss cone is large even at the lowest values of B sampled at each $L$. Around $L=1.9$, the minimum $B$ sampled during the period in Figure 1 is 0.20 gauss. Fluxes observed just a little way down the field line, at $\mathrm{B}=0.22$ gauss, are about $60 \%$ lower; at $\mathrm{B}=0.24$ gauss, the flux is less than $5 \%$ of its value at 0.20 gauss. Taking this value of $B$ to define the limit of the drift-loss cone gives a $66^{\circ}$ pitch angle as its edge at $\mathrm{B}=0.20$ gauss; the cosine of the equatorial pitch angle of this edge is then $x=0.90$, in agreement with the calculations by Walt (1966) of drift-averaged atmospheric losses at $\mathrm{L}=1.9$. The minimum $B$ sampled at each $L$ in each of the five time periods varies slightly, but, in each case, it is no more than 0.21 gauss; thus, we can measure the decay of the electron flux by comparing the intensity in the five time periods at 0.21 gauss or above. Figure 2 shows the average from $\mathrm{L}=1.78$ to 2.02 and $B=0.21$ to 0.22 gauss of the sum of the counting rates of Deep Range electrons. (The data were rebinned with ten-fold finer resolution in B before averaging in order to correct for differences in sampling of B from period to period, and systematic errors were estimated from the scatter among the points in each

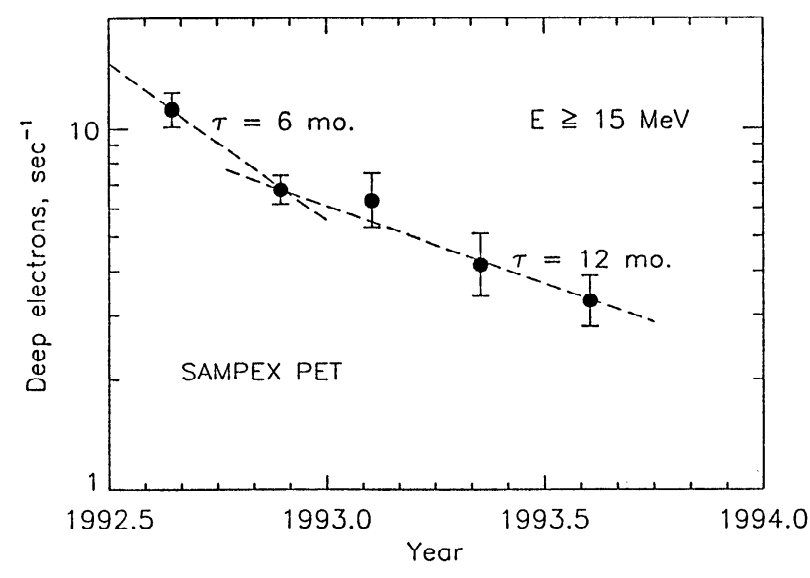

Figure 2. Summed Deep Range electron counting rates perpendicular to the magnetic field at $\mathrm{B}=0.21$ to 0.22 gauss as a function of time, averaged across the peak in L. Dashed lines indicate decay curves for two values of the $e$-folding lifetime.

average.) The $e$-folding time of the decay varies from six to twelve months; however, because of our coarse sampling in time and the increasing systematic errors, we are not able to fix the time of the apparent change in the decay constant well enough to identify a causative magnetospheric event in other observations.

\section{Energy Spectrum}

Due to the failure of the P47 ADC, we cannot directly measure the energies of particles stopping in P4 through P7, and the range straggling of electrons makes it impossible to convert penetration depth to energy on an individual particle basis. We can, however, use the penetration depth in a statistical manner, provided we can model the response of each Range to incident electrons of different energies. This was done using the EGS4 electromagnetic shower Monte Carlo simulation code (Nelson et al. 1985); the results are shown in Figure 3. The response plotted is the effective geometry factor of each

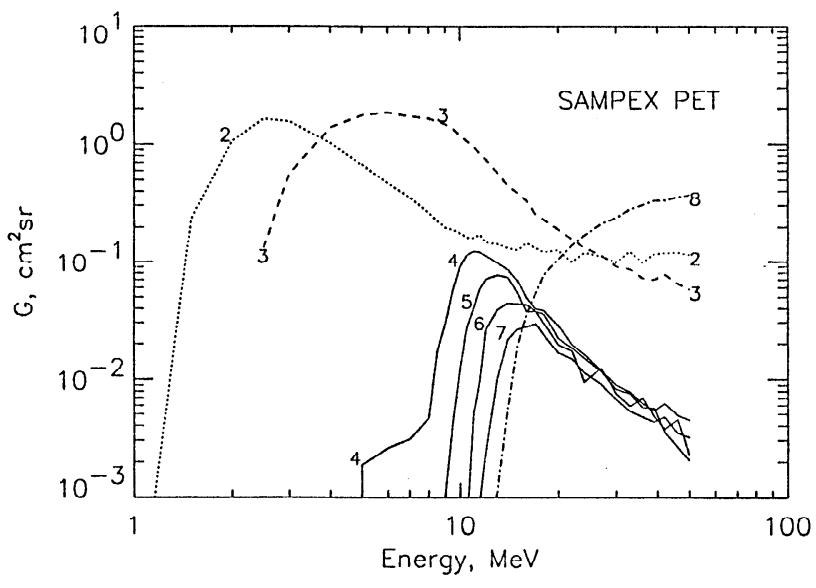

Figure 3. Effective geometry factor of PET electron Range rates as a function of energy for isotropic incidence. (In the observed pancake pitch-angle distribution, relative responses of Ranges 4 to 8 are the same, with normalization about $40 \%$ lower.) Numbers label Ranges, and dotted and/or dashed lines are as in Figure 1. 
Range's counting rate for an isotropic flux of monoenergetic electrons. Numbers label the different ranges, and the dotted and/or dashed lines correspond to the same Ranges as in Figure 1. Features to note in Figure 3 are the close similarity between the geometric factors of Ranges 4 to 7 above about $16 \mathrm{MeV}$ and the substantially different geometric factors of these Ranges below $16 \mathrm{MeV}$ down to their thresholds. Also noteworthy is the much greater geometric factor of Range 8 starting above 16 $\mathrm{MeV}$. Since the observed counting rates of the Deep Ranges are so similar ( $c f$. Figure 1), with the Range-7 response just slightly below that of Range 4, we can conclude that only a small fraction of the electrons have energies between $\sim 8$ and $15 \mathrm{MeV}$. Otherwise there would have been a large differential response in Ranges 4 to 7 . On the other hand, if a substantial fraction of the electrons had energies above $-20 \mathrm{MeV}$, we would have seen a very strong response in the penetrating rate, Range 8.

These expectations are illustrated in Figure 4, where the different Ranges' responses relative to that of Range 7 are plotted for a family of spectra similar to that of the electrons injected in the March 1991 event, which had a power law with an index of -6 and a lower cutoff of $15 \mathrm{MeV}$. Here we allow the cutoff to vary while the slope is held fixed. In this Figure, we see that the Range- 4 through -7 rates converge as seen in Figure 1 when the cutoff rises above about $15 \mathrm{MeV}$; this conclusion is independent of the spectral index. The range of cutoff energies labeled " $A$ " in Figure 4 shows where the response of Range 4 is between 1.7 and 2 times that of Range 7, approximating the values in Figure 1. We also see that the ratio of the summed Deep Rates (labeled $\Sigma 4-7$ ) to the Range-8 rate approximates that in Figure 1 at $\mathrm{L}=1.9$ for a cutoff around $15 \mathrm{MeV}$; the range of cutoff energies labeled " $B$ " in the Figure shows where this ratio is between 1.5 and 2 . This ratio falls with a hardening spectral slope as well, so we may assert that the spectrum falls nearly as steeply as was observed during the injection event. However, further analysis of background is needed to understand the Range- 8 rate before we draw conclusions about the exact spectral shape. We could also consider the Range- 3 rate in this analysis, but since the response of this Range at these energies is quite broad and roughly parallel to those of the Deep Ranges, its inclusion would not impose much additional con-

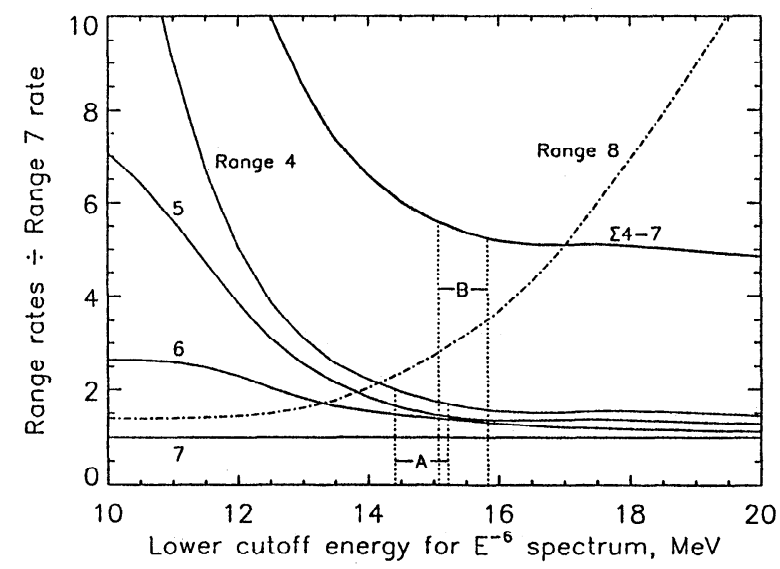

Figure 4. Responses of PET Ranges 4 to 8 , relative to Range 7 for a family of electron spectra with $E^{-6}$ slope and varying lower cutoff energies. Regions labeled " $A$ " and " $B$ " show observational constraints on the cutoff, as described in the text. straint on the spectrum. In addition, the background in this Range from lower-energy inner-zone electrons is uncertain in the vicinity of the peak. The above analysis of Ranges 4 to 8, then, allows us to attribute the peak around $\mathrm{L}=1.9$ to electrons with a steeply falling spectrum starting at about $15 \mathrm{MeV}$, as expected for the remnants of the electrons injected in March 1991.

\section{Discussion and Conclusions}

Although the SAMPEX measurements do not permit exact derivation of the electron energy spectrum, they do tightly constrain the possibilities, as detailed above. The observations are entirely consistent with the electrons injected by the shock of 24 March 1991 having closely maintained their injected energies while declining in intensity. Clearly, flux losses predominate over energy losses. It appears that electrons are pitch-angle scattered until their mirror points reach B $\approx 0.24$ gauss, below which they are quickly removed. The apparent inward motion of the peak intensity, about 0.05 units in $\mathrm{L}$ over the period discussed here, suggests that some radial diffusion is still occurring. The CRRES data (Blake et al. 1992b) showed that soon after the electron injection event, some radial diffusion into the region below $\mathrm{L}=2$ did occur. Also, the pitch-angle distribution flattened greatly, and electrons injected above $\mathrm{L} \approx 2.5$ disappeared relatively quickly. These CRRES observations are interpreted as indicating that wave-particle interactions rapidly scattered and removed electrons at the higher $\mathrm{L}$ values. Radial motion of the electrons left at lower $L$ values did not lead to significant energization as a consequence of the small radial distance moved. These changes in the radial and pitch-angle distribution also preclude a direct comparison of the lifetimes in Figure 2, measured for a nearly constant distribution in $\mathrm{L}$ and pitch angle, with the measurements from CRRES nearer the time of the belt's formation.

The lifetime of electrons trapped around $L=2$ and with energies $>0.5 \mathrm{MeV}$ was derived earlier from observations of fission electrons. This lifetime is $\sim 6$ months ( $c f$. Roberts 1969), which is much the same as our result for $15-20 \mathrm{McV}$ electrons. To understand this, we extrapolated to higher energies the calculations by Lyons et al. (1972) of electron lifetimes against pitch-angle diffusion into the loss cone. Their work showed that resonant interaction with ambient whistler-mode turbulence in the plasmasphere results in lifetimes with a minimum value at an energy that rises with decreasing $L$, as shown in Figure 5. Their calculations of electron lifetimes are shown as small open circles, and the minimum of a parabola (dotted line) drawn through the three shortest lifetimes at each $\mathrm{L}$ is plotted as a large filled circle. Most of the curve for $L=2$ is above the 2-MeV limit of their calculations. However, the energy at the extrapolated minimum (shown as a large open circle) fits their expected $\mathrm{L}^{-5}$ dependence well, so we may use the curve to predict that the lifetimes in the fission electron energy range (below the minimum-lifetime energy) and for the shockinjected electrons discussed here (above the minimum) should be similar, as observed. The lifetime from their calculations in both cases is $-3-4$ months; however, they assumed a rather high amplitude for the whistler noise and the lifetimes scale as the inverse square of this amplitude, independent of energy. Adopting a value of noise amplitude closer to that used for average magnetospheric conditions $(10 \mathrm{~m} \gamma)$ by Lyons and Thorne (1973), we can bring theory and observation into better agreement. 


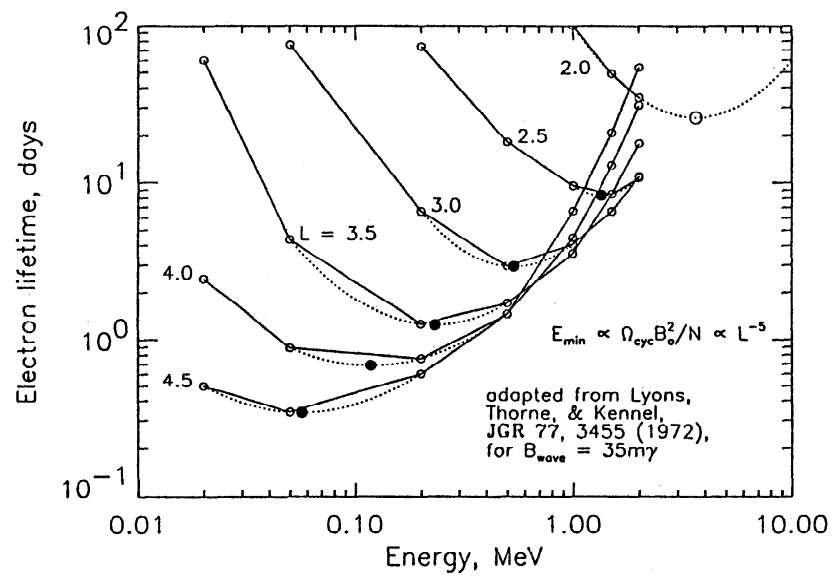

Figure 5. Calculated electron lifetimes, adapted from Figure 7 of Lyons et al. (1972), with parabolic extrapolation to higher energies at $L=2$. Numbers label values of $L$; actual calculations are shown as small open circles connected by solid lines.

Acknowledgments. This work was supported by NASA at The Aerospace Corporation under NASA Cooperative Agreement 26979B and at the California Institute of Technology under contract NAS5-30704 and grant NAGW1919. One of us (MDL) was supported by an NRC Research Associateship. The authors would like to acknowledge several discussions with Larry Lyons and Mike Schulz on the lifetimes of radiation-belt energetic electrons, and with Richard Selesnick on the calibration of PET.

\section{References}

Baker, D. N., G. M. Mason, O. Figueroa, G. Colon, J. G. Watzin, and R. M. Aleman, An overview of the Solar, Anomalous, and Magnetospheric Particle Explorer (SAMPEX) mission, IEEE Trans. Geosciences and Remote Sensing, 31, 531, 1993.
Blake, J. B., W. A. Kolasinski, R. W. Fillius, and E. G. Mullen, Injection of electrons and protons with energies of tens of $\mathrm{MeV}$ into $\mathrm{L}<3$ on 24 March 1991, Geophys. Res. Lett., 19, 821, 1992a.

Blake, J. B., M. S. Gussenhoven, E. G. Mullen, and R. W. Fillius, Identification of an unexpected space radiation hazard, Proceedings IEEE Trans. Nucl. Sci., 39, 1761, 1992b.

Cook, W. R., A. C. Cummings, J. R. Cummings, T. L. Garrard, B. Kecman, R. A. Mewaldt, R. S. Selesnick, E. C. Stone, D. N. Baker, T. T. von Rosenvinge, J. B. Blake, and L. B. Callis, PET: A Proton/Electron Telescope for Studies of Magnetospheric, Solar, and Galactic Particles, IEEE Trans. Geosciences and Remote Sensing, 31, 565, 1993.

Lyons, L. R., and R. M. Thorne, Equilibrium Structure of Radiation Belt Electrons, J. of Geophys. Res., 78, 2142, 1973.

Lyons, L. R., R. M. Thorne, and C. F. Kennel, Pitch-Angle Diffusion of Radiation Belt Electrons within the Plasmasphere, J. of Geophys. Res., 77, 3455, 1972.

Nelson, W. R., H. Hirayama, and D. W. O. Rogers, The EGS4 code system, SLAC Report 265, 1985.

Roberts, C. S., Pitch-angle diffusion of electrons in the magnetosphere, Rev. Geophys. 7, 305, 1969.

Vampola, A. L., and A. Korth, Electron drift echoes in the inner magnetosphere, Geophys. Res. Lett., 19, 625,1992.

Walt, M., Loss rates of trapped electrons by atmospheric collisions, in Radiation Trapped in the Earth's Magnetic Field, p. 337, ed. B. M. McCormac (Dordrecht, Holland: D. Reidel, 1966).

M. D. Looper, Space and Environment Technology Center, The Aerospace Corporation, M2-260, P. O. Box 92957, Los Angeles, CA 90009. DIRAC2::LOOPER

J. B. Blake, Space and Environment Technology Center, The Aerospace Corporation, M2-260, P. O. Box 92957, Los Angeles, CA 90009. DIRAC2::BLAKE

R. A. Mewaldt, California Institute of Technology, Pasadena, CA 91125. CITSRL::DICK

J. R. Cummings, California Institute of Technology, Pasadena, CA 91125. CITSRL::JRC

D. N.. Baker, University of Colorado, LASP, Boulder, CO 80309. ZODIAC::BAKER

(Received: February 25, 1994; revised: May 19, 1994; accepted: June 8, 1994.) 